$\mathbb{T}$ periodica polytechnica

Mechanical Engineering

$52 / 1(2008)[25+33$

doi: 10.3311/pp.me.2008-1.05

web: http://www.pp.bme.hu/me

(C) Periodica Polytechnica 2008

RESEARCH ARTICLE

\section{Human Weaknesses and strengths in collaboration with robots}

Olessia Ogorodnikova

Received 2007-07-17

\begin{abstract}
This paper describes the importance of the incorporation of ergonomics, human factor, errors analysis and cognitive engineering approaches in the design of human-robot systems, how consideration of these subjects help designers and workers to avoid hazardous situations and make human-robot interaction in vicinity more effective, reliable and safe. Basing on acquired knowledge and guiding by acquired knowledg e we propose our trial application for disassembly cell.
\end{abstract}

\section{Keywords}

Robotics $\cdot$ human factor $\cdot$ cognition $\cdot$ ergonomics

Olessia Ogorodnikova

Department of Mechatronics, Optics and Instrumentation Technology, BME e-mail: olessia@git.bme.hu

\section{Introduction}

The domain of Human Factors was scrutinized in Air Traffic Control, Cockpit Design, Nuclear Power plants, and Chemical Processing plants research. While these areas differ from robotics, there are many theories and results related to human workload, vigilance, situation awareness, human error, ergonomics and cognitive engineering that can also be applied to HRI development. For instance, NASA provided documentation which contains quantitative information about human body size, posture, movements, surface area, acceptable noise, vibrations, radiation, illumination, thermal levels, etc. for NASA crewmembers. Human Reliability and different safety analysis for Nuclear Power plant were considered in D. Gertman's and $H$. Blackman's work. [1] In the robotic field, human errors have to be a primary concern as well. A lot of work has been done with regard to direct robot human interaction and the dangers therein to the human from the robot [2], but very little work has studied the robotic system's failure being caused by the human. Though, it is well known that about $85 \%$ of accidents were caused by human errors, and in $50 \%$ cases by improperly designed robotic workstations. Both areas are important and require more focus. In this paper, besides the issue of the human factor, there will be discussed the aspects of ergonomics and cognitive engineering as inevitable parts of the scenario: safe, reliable, effective human centered robotic work cell design.

The areas of discussion include:

- Cognitive Engineering

- Human Error

- The question of Ergonomics

\section{HCD and cognitive engineering (Robotics)}

The general principle of Human Centered Design (HCD) is that the Human plays an integral role in the system design, development, testing, participating, etc. Humans may represent different roles: supervisor, operator, maintenance, teaching and observer. [3] Depending on the particular role in every interaction, there are many different ways to determine with which 
extent of safety, reliability and effectiveness each Human Robot Interaction System could be defined as a Quintuple [4]:

$$
H R I S=(T, U, R, E, I)
$$

where $T$ - task requirements (cognitive and physical), $U=$ (UC, OP) - user characteristics: $U C$ - cognitive, $U P$ - physical, $R=$ (RS, RH) - robot characteristics (soft-, hardware), $E=$ environment, $I=$ a set of interactions.

Robots are capable of high speed, accuracy, a wide range of movements as well as power, whereas humans are slow, with limited capabilities, high sensitivity to the ambient environment and inclined to commit errors frequently (to err is but human). On the other hand, the human is much more flexible and adaptable in thinking, motion, perception and behavior. The combination and Integration of advantages of both humans and robots and the incorporation of them into a hybrid co-operational system would definitely improve efficiency of performance. However, we need to know what the characteristics that would have significant influence are. For a better understanding of human behavior in different situations, we should turn to cognitive engineering and psychology. Cognitive engineering is defined as "joining the cognitive sciences with engineering to form a new, applied discipline for the design and construction of systems to support the human". [5] Cognitive engineering can be described as an approach to the design of better cooperation of human and technology, whereas cognitive robotic can be similarly described as the design of better interaction between humans and robots. The basic tenant of cognitive robotics is that it will enhance human-robot interfaces if they are designed taking human's cognitive information processing, decision making, perceiving and others capabilities or limitations into account. Below we consider some of these which play a significant role in robot-human collaboration.

\subsection{Information processing}

A large number of studies show that in unaided conditions humans are highly inadequate at number of information processing tasks. The presence of a great amount of unstructured information may make performance more erratic and poorer and is associated with cognitive overload. In information processing, the approach to understanding human behavior relies upon the fact that there is a limit to the number of mental processing operations that can be carried out at any one time. Thus, overload, omissions, commissions, task shedding are all expected consequences of exceeding the information capacity of individuals. For example, stimuli can appear so closely together in time that they exceed the channel capacity for a particular modality such as vision or audition; poor temporal cues or poor intensity cues may result in poor performance. It is also recognized that higher level cognitive processes play a significant role in high technology mishaps [6]. The human's ability to perform the crucial mental activities and perform tasks effectively rests upon fundamental cognitive processes and functions. There are 3 ma- jor information-processing stages: perception, decision and response execution. (Fig. 1)

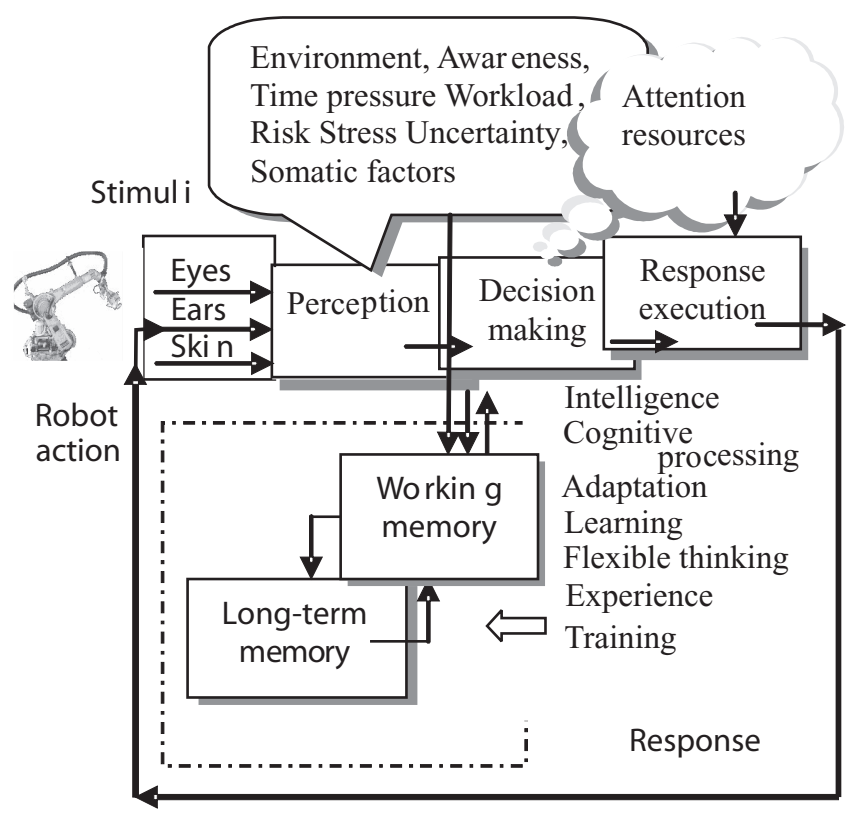

Fig. 1. Model of Human Information Processing.

\subsection{Perception and Decision-making (DM)}

Humans receive the information visually or via an auditory organ (perception), understand and interpret the meaning of the perceived information and make decisions interacting with the knowledge stored in the memory system (cognition). There are various levels of perception that depend on the stimulus and the task confronting a person. The most basic form of perception is simple detection the more complicated are identification and recognition. The act of perception involves our prior experiences and learned associations. Even the act of simple detection involves some complex information processing and decision making. Limits of human performance could appear from the required time, the amount of information that should be processed per unit time, complicity, etc. Two decision-making characteristics are especially important: how much time decision-making requires and how accurate decisions are. The rate of information flow per unit time remains constant at about $1 \mathrm{bit} / 220 \mathrm{msec}$. However, if the operator exceeds these margins by trying to go too fast, accuracy drops very rapidly and the rate of information transmission will fall. [7] Humans make hundreds, if not thousands of decisions every day. These decisions are made rapidly in dynamic environments under varying conditions. Depending upon the human's current task, such decisions may have dire consequences if incorrectly determined. Another important characteristic is mental workload - difference between the amount of resources available within a person and the amount of resources demanded by the task. In robot-human cooperation systems, we must perform many kinds of tasks using complicated cognitive information processing. Excessive workload or fatigue reduces the efficiency and induces more errors. There- 
fore, an effective method for monitoring mental workload or fatigue would be a useful tool for the evaluation of alternative robot-human cooperative systems. The design must be such that an acceptable level of work load and job satisfaction is maintained. Sanders and McCormick [8] define mental workload as: "a measurable quantity of the information processing demands placed on an individual by a task." Each human has a different mental processing capacity. This mental capacity may be affected by lack of tasks to complete, stress, lack of sleep, environmental conditions, and even missing information. The more stressful the particular situation is the more likely that the operator's mental workload will be very high. That is why, during human-robot interface planning we should thoroughly think over every operation, amount of data and information flow on the whole bearing on humans in every unit of time.

\subsection{Awareness and Vigilance}

Vigilance is "sustained attention, signals, detection, staying alert, being able to identity targets, and maintaining performance over time" [9]. Maintaining vigilance can be affected by many factors. It has been found that lack of sleep [10] as well as circadian rhythms may adversely affect it. Also environmental factors, such as: lighting, noise, ventilation, vibration, and temperature, as well as too much information and/or too many tasks that operator should work out simultaneously often influence the vigilance level. Another important aspect in Human Factor studies is awareness - knowledge of what is going on around you. Experiments by Gugerty and qemphTirre [11] show that situational awareness is correlated with working memory, perceptual-motor ability, static visual processing, dynamic visual processing, and temporal processing ability. In addition, studies have shown that the ability to acquire situational awareness decreases with age. The SAGAT tool has been used to show that there is a decrease in situational awareness with fully automated systems. [12] Goodrich, Olsen, Crandall, and Palmer [13] introduced the concept of neglect to capture the relationship between user attention and robot autonomy. The idea is that a robot's effectiveness decreases as the operator fails to attend to that robot. Neglect can be caused by time delays in remote operations or by increased workload on the part of the operator.

There are three levels of awareness [14]:

- The basic perception of cues. Failure to perceive information can be due to the short comings of a system or they can be due to a user's cognitive failures.

- The ability to comprehend or to integrate multiple pieces of information and determine the relevance to the goals the user wants to achieve.

- The ability to forecast future situation events and dynamics based on human's perception and comprehension of the present situation.
A person can fail to perform correctly due to poorly designed systems or due to cognitive failures. During the collaboration with robots humans are very likely to misperceive some hazards related to the robot's movements. For instance, they can misunderstand the robot's state, underestimate or overlook the danger zone, fail to respond to a recognized hazard, misperceive the direction of robot arm movement etc. Owing to inadequate equipment (control, pendants) or workplace design they may have difficulties to specify functions, procedures, confuse buttons, etc. Primarily, humans should be aware of the current status of the robot, what action it is carrying out at this moment and what it plans to do next. Special training should be conducted according to each interaction with the robot, external awareness system should be installed (indicators, signals, sensors). User interface also must be supplied with some visual, auditory, tactile feedback providing clarity and unambiguity in operational instructions, be easy to use, not complicate the task, promote learning, be reliable and have a standardized layout.

\section{Human Error}

J. Reason defines error as "... all those occasions in which a planned sequence of mental or physical activities fails to achieve its intended outcome, and when these failures cannot be attributed to the intervention of some chance agency" [6]. Sanders and McCormick define human error as "an inappropriate or undesirable human decision or behavior that reduces, or has the potential of reducing effectiveness, safety, or system performance" [8]. Very often errors are the result of faulty cognition. For cognitively complex tasks, it may be possible to classify errors according to the different stages of information processing (Fig. 2), thereby differentiating errors related to perception from errors related to failures in working memory. Very simple error taxonomy defines errors of omission (forgetting to or something) and commission (doing something incorrectly). The last one is often categorized as errors related to sequence, timing and substitution. From experimental studies in neurocognitive psychology aiming at investigating cognitive processes such as perception, working memory and decision making it is known that humans are able to split and allocate processing capacities to different tasks at the same time by applying control processes that generate a hierarchical sequencing order. However, although humans are quite successful in perceiving and doing multiple things at the same time, there is a probability of task not to be accomplished properly. Potential sources of performance errors may, e.g., be due to interference stemming from tasks that have to be performed simultaneously or from interference caused by transfer or crossover effects from previous activity. Rasmussen [15] proposed a cognitive engineering approach to better understand the human's information processing and human-machine interaction. He classified human behavior into: skill-, rule-, and knowledge-based levels. The skill-based behavior represents sensorimotor performance during activities that take place without conscious control as smooth, automated, 
and highly integrated patterns of behavior. Mistakes are the result of misinterpretation or misunderstanding. The rule-based level is based on explicit know-how, and we can consciously report the rule. Rules may be misapplied in a variety of situations (diagnosis) Mistakes occur when one applies the wrong rule or applies a correct rule at the wrong time. Knowledge-base behavior occurs when the environment lacks supporting external cues: procedures, signs, or other types of displays that aid in making decisions. For instance, if there is unclear or misleading systems feedback, lack of control indication, false or misleading procedures, inexperience or unavailability of systems. Errors can be directly attributed to technical system design, environmental, and personnel factors. While the technical system design can be controlled to eliminate and/or reduce human error occurrence, the control of environmental factors and the way the system is used by personnel is often less controllable. The Figure 3 illustrates the information processing steps involved in an accident sequence and lists factors that affect each stage of the process. Each step in this sequence can lead to accident and human injuries, and our task, as designers, is to predetermine all possible errors, failures and misuses and eliminate their occurrence or reduce the extent of dangerous impact. The causes of errors can be various, but, if we look back at Rasmussen's taxonomy, the grounds might emerge from: lack of knowledge of the standard, requirement or need (KB); lack of the appropriate level of cognition: ability to understand, apply, analyze, synthesize (cognition-based SB); lack of judgment in evaluating risk versus benefit; lack of behavioral techniques for conservative decision-making (decision-based); lack of dexterity (SB), etc. There are a number of system safety techniques that can be utilized for proactive investigations of potential risk in systems to maximize reliability as well as for retrospective accident investigations. The most frequently used technique for Human failure analysis is the so-called HRA Event Trees which support event sequence logic structures including cognitive activity and corresponding errors associated with human performance. The cognitive approach provides the means to understand behavioral mechanisms and discusses the intellectual activities associated with human performance. Typically the branches represent a binary decision path in which correct and incorrect performance are the two choices. Analysts detect a failure rate associated with incorrect performance and place that value on the right hand side of the tree. [1] Fig. 4 represents the model of this tree.

The probabilities of erroneous or correct actions can be computed in the following way:

$$
\begin{aligned}
& P_{s}=a * b * c \\
& P_{e}=A+a * B+a * b * C
\end{aligned}
$$

where $P_{s}$ and $P_{e}$ - the probabilities of success and error correspondingly.

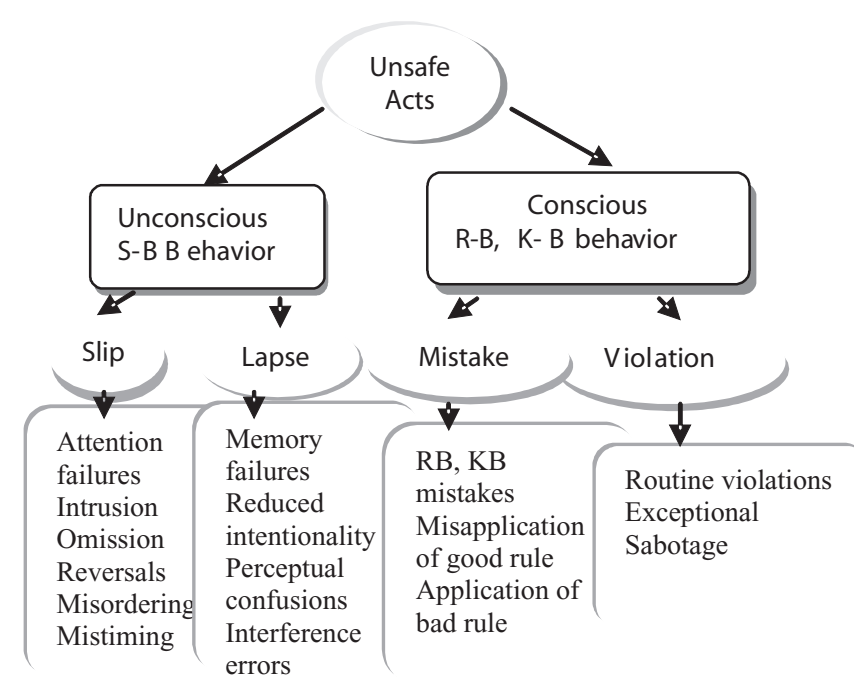

Fig. 2. Human error taxonomy.

\section{The Role of Ergonomics}

The ergonomic guidelines and principles are meant to provide an orientation towards the physiological needs of the operator. The design is essentially a compromise between human biological and physiological needs, as determined by the ergonomic guidelines and the physical, safety requirements of the workstation in terms of the size, functions, frequency and methods of use of the individual equipment or of interactions with robots. Uppermost, the ergonomic design should minimize the potential Human error. However, we cannot just memorize and follow the set of ergonomic rules or applying a generic checklist to a workplace situation. We should also develop proper biomechanical reasoning when assessing the physical demands of a workplace. Various biomechanical concepts must be considered collectively. The most important concepts that should be predefined before humans start collaborating with robots are imposed magnitude of the load (task, force shearing), mutual allocation, dimensions, safe methods of operation (vibration, impact forces, speed, postures, etc.) A fundamental concept in the application of occupational ergonomics is that workplace must be designed so that the load imposed on a body structure does not exceed the tolerance of the structure. When we apply ergonomic rules into robotics we consider human centered work place with respect to robot specific physical characteristics and optimize the work without any threat to human's health providing still efficiency of the performance. The effective task sharing between Humans and robots will definitely release humans from excessive overloads (mental, muscular). However, humans and robots have advantages and disadvantages in performing the task, and it is important to evaluate all task related capabilities and limitations of partners. For instance, S. Nof [17] developed RobotHuman charts to help determine whether a robot or human can perform a given task. It is composed of three main types of work characteristics, i.e. (1) physical skills, (2) mental and communicative characteristics, (3) energy considerations. The first type 


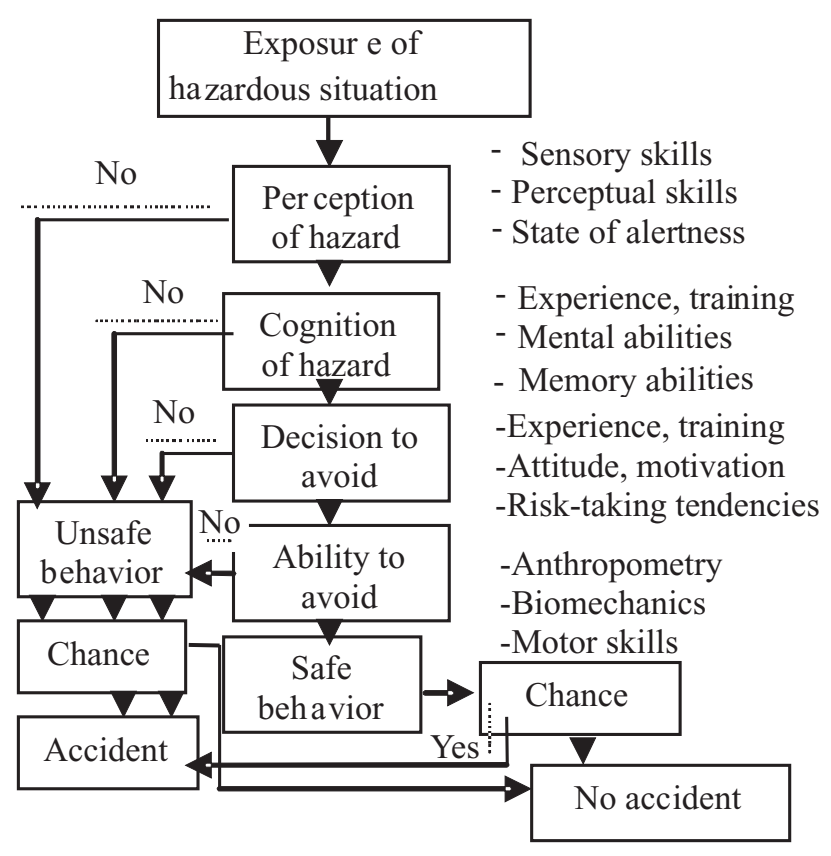

Fig. 3. Accident sequence model.

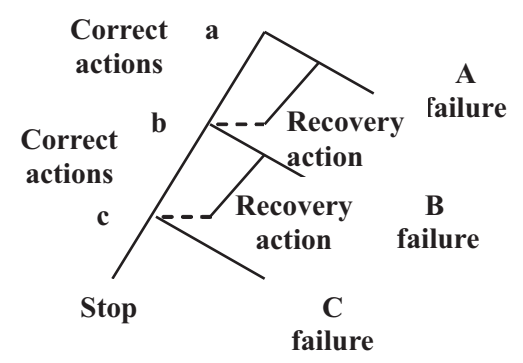

Fig. 4. HRA Event Tree.

of characteristics includes consideration of manipulation, body dimensions, strength and power, consistency, overload performance, and environmental constrains. The second chart refers to mental and communicative skills, while the third one presents a comparison of robot and human energy and power characteristics. It is also true that when introducing robots into the human environment conventional occupation ergonomics is not sufficient. Thus, besides considering the human's measurements (anthropometry, physiology, and biomechanical characteristics) now we should also take into account the robot's anatomy, mechanics, dynamics, skills and work abilities to optimize the mutual work making it more. Working in close vicinity with robots means a high probability of contact that can cause pain and injuries to the human body. Therefore, it is essential to know the human's tolerance to these stimuli and to design the $\mathrm{h}-\mathrm{r}$ coexistent system with this consideration. Several experiments were conducted to gain these limitations [18]. It was defined that the parameters of the moving speed of robots, acceleration, distance from the human, and the size of contact area are have a great impact on the tolerance magnitude. Several categories of pain are believed to exist that might be used as tolerance limits in work place design: structural disruption, tissue stimulation, physio- logic limits and psychophysical acceptance. Each of these pathways is expected to respond differently to physical impacts. It was proposed [18] to set the contact safe force equal to $50 \mathrm{H}$ if the contact area is less then $18 \mathrm{~mm}$. However it is still a great challenge to measure a person's tolerance in each part of the body while they have different levels of sensation.

\subsection{Human constrains, capabilities, attitudes}

There are some reports on the examination of the effects on the psychological side of human beings exerted by spatial movements of robots and on human reaction induced by robot movements. Those include the effect of distance to a robot, the effect of the moving speed of the robot, and the effect of the moving acceleration of the robot. Humans should be able to perceive, predict, and react to a robot's arm movements in time, to stop the arm before an accident or injury can occur. It is therefore important that the speed of the robot is not too high and that changes in movement pattern are predictable. US standard ANSI/RIA [20] states that a slow speed of $250 \mathrm{~mm} / \mathrm{s}$ must be the maximum initiated at the teach pendant. On the other hand, the National Safety Council recommends that robot movement should be limited to $150 \mathrm{~mm} / \mathrm{s}$. Experimental results conducted by several researches (See Fig 4) were also controversial. Trials provided by Nagamachi [21] showed that subjects felt safe placing themselves within $225 \mathrm{~mm}$ from the robot if it moved at the speed $250 \mathrm{~mm} / \mathrm{s}$. In view of the results the optimal operational speed was recommended as $300 \mathrm{~mm} / \mathrm{s}$. Relevant experiments were provided by [16] Sugimoto, where the safe robot speed was chosen as 140 $\mathrm{mm} / \mathrm{s}$ with a corresponding safe distance $200 \mathrm{~mm}$. In work conducted by Etherton [22], if robot speed was $250 \mathrm{~mm} / \mathrm{s}$ human responses were defined with a mean overrun distance of 77,7 $\mathrm{mm}$ and $109 \mathrm{~mm}$ at the average velocity $450 \mathrm{~mm} / \mathrm{s}$. Additionally it was found that the reaction time (RT) on robot's movements were slower if the speed was decreased and varied in the range of 0,3-1,5 s. Respond on slower robot arm speeds seems to include decision-making component which increases the RT, in this case humans give the robot their full attention and less error takes place. Whereas as a robot arm speed increases there is a progressively less of a decision cost component, reaction is more reflexive and thereby more erroneous. The maximum RT under ideal conditions is found as $1.4 \mathrm{~s}(0,5 \mathrm{~s}$ plus two standard deviation) when robot would move $19,9 \mathrm{~cm}$ if the speed is 140 $\mathrm{mm} / \mathrm{s}$ [22]. This time we need to anticipate hazard: perceive, cognate and react in a proper way. However, RT increases with a number of alternatives (about $150 \mathrm{~ms}$ ). If different stimuli occur with different probabilities time will be shorter for the more probable ones because expected signal has already appropriate response in our memory and can be retrieved very fast. If signals appear at the same time and demand different response, there is latency between and also increase in RT. So when we deal with a safety in robotics, when we need to alert human about danger as quick as possible, we should plan the warning system so that signals would complement each other generating unambiguous 
stimuli for human could make an appropriate response. These stimuli we call "active awareness stimuli" which suppose to enhance human alertness and strengthen their sensory capability. The main sense of human is vision. It is defined that between 20-30 mm human vision is optimized, receives a better sense of control under robot. However, stimuli presented in the peripheral field of view ( $45^{\prime}$ from fovea) are responded to about 15-30 ms slower then are centrally presented stimuli. The relationship between visual attention and working memory implies a potential competition among visual attention and other cognitive tasks. Additional sensory cues might improve or aggravate the attention. It is should be noted, time for visual processing is comparatively large (slower than RT on auditory and tactile stimuli with $40 \mathrm{~ms}$ ). So as a consequence aiming at improve human awareness and augment human sensitivity to stimuli we need to compensate visual cues with complementary ones (tactile, auditory) but without any suppression. Some research states that people's attitudes toward robots depend on their personality. For instance, extraverts are more likely to approach near to robots, and tend to come extremely close to them ignoring safety standards and committing risk taking actions. There are also people who feel anxiety about robots having mental resistance to their applications that prevents individuals from interaction with them [23]. Various experiments showed that front right and front left approach directions to robots were rated as the most comfortable, while rear approaches were rated as the least comfortable, that humans prefer interact with robots from the side positions when the movements are smooth, unlinear, and more human-like; when the velocity peak locates at the front position in the movement time. Drawing a parallel with a Human-human interaction we can easily define that the distance that people prefer to keep from the robot equal to those that they usually hold interacting with unknown person, not too close, distance enough for auditory conversation (personal space, 0,5-0,9 m). However if there is a necessity in physical contact this distance can be diminished to $20-30 \mathrm{~cm}$. We suggested that the most comfortable and safe (human has time to react and move away in the case of danger) distance is $90-20 \mathrm{~cm}$, while the most dangerous and unwilling is under $8 \mathrm{~cm}$. Here the radius formed by the extended hand, while moving over the working surface centered at the shoulder joint, and the magnitude can be determined as in Equation (4) [8]:

$$
R=\sqrt{\left(K^{2}-(E-L)^{2}\right)}
$$

where $\mathrm{R}$ - extended arm radius, cm; $\mathrm{K}$-arm length, cm; E - shoulder height $\mathrm{cm}$, and $\mathrm{L}=$ elbow height, $\mathrm{cm}$. For the safety distance (See Eq.4) we consider human's waking speed, speed of movements, human RT, stopping time of the robot and responding time of the safety system.

$$
D_{s}=\left(V_{w}+V_{m}\right) T_{h}+V_{r}\left(T_{s r}+T_{s c}+T_{r s}\right)
$$

where $V_{w}, V_{m}, V_{r}$ - walking velocity, speed of movement and robot operational speeds correspondingly; $T_{h}$ - human reaction time, $T_{s r}, T_{r s}, T_{r s}$ - robot stopping, control system and sensory respond time correspondingly. Area of convenient reach requires less forward extension of the forearm minimize stress. For instance, distance with $39.4 \mathrm{~cm}$ will be conveniently reached with a sweep forearm while the upper arm hangs in a natural position. Functional arm reach depends on direction, nature of activity, use of restrains, age, sex, handicaps. Moreover, due to physical structures motion can be made more rapidly in certain directions and this time is a logarithmic function of the distance. Taking into account factors mentioned above it becomes obvious that psychological and physiological aspects have a significant influence on the design and should be definitely considered within the interactions planning stage. However this topic still requires more studies, scientific proves and standardization.

\subsection{Methods of measurements}

There are three main methods of evaluation human capabilities and attitudes used for human-robot interaction (HRI) studies [24]: self-report measures, behavioral measures and physiological measures. Self-report measures include computerized psychometric scales, questionnaires, and/or surveys. Behavioral measures are often included in psychophysilogical evaluations for convergent validity of participants' self-report responses and measured physiological reactions. Psychophysiology focuses on the interaction between the mind and body and the most common measures in Human-Robot Interaction studies include: cardiovascular system (heart rate variability (HRV), respiratory sinus arrhythmia (RSA), cardiac output, interbeat interval (IBI), blood pressure (BP)); electrodermal activity (skin conductance activity (SCA), skin conductance response (SCR)); respiratory system (breaths per minute, respiration volume); muscular system (electromyography (EMG)); and brain activity (electroencephalography (EEG) and imaging). For instance, neurocognitive methods [25] include behavioral measurements, EEG recordings and ERP analysis, Motion and Eye tracking. In the field of neuroergonomics, ERPs have proven to be useful for the investigation of mental workload. ERPs are small voltage fluctuations measured at the human scalp that reflect neural activity that can be associated with sensory, motor or other cognitive processes. ERPs are extracted from the spontaneous brain activity (the EEG) by special averaging procedures taking advantage of the fact that ERPs are time-locked to the event (perceptual, cognitive, or motor) they are related to. Motion tracking systems have often been employed in ergonomics research, e.g. in the investigation of complex work environments that demand effective human control, predictions, and decisions in the presence of uncertainties and unforeseen changes in work system parameters. Eye tracking has been a powerful tool in cognitive neuroscience to investigate the cognitive and neural mechanisms that govern eye movements. In ergonomic research, eye tracking has been used to identify non-optimal aspects of human-robot interface. In particular, although there is no strict relation between the focus of gaze and the focus of at- 
tention, the pattern of gaze shifts may be taken as valuable predictor of the operator's focus of attention. For example, it could be investigated if and how salient events (e.g. warning signals) may attract or distract an observer's attention. These methods enhance the understanding of how humans and automated machines interact and how this interaction could be improved. Results help to design evaluate constraints of human perception, processing capacities and cognitive control and thus error rates can be decreased, work optimized and safety provided. For instance, we can use derived information to improve warning system design and enhance human awareness about ambient environment (robot movements, task). Each of the methods can be regarded optimal in addressing certain aspects, however, only the combination of these methods will give the most comprehensive insights. Depending on the specific research questions, different combinations of these measures may be used.

\subsection{Guidance of General safety design}

The designer should remember that most errors and safety problems are the result of the equipment not being designed properly or being used improperly. The designer must anticipate how equipment might be misused and design it so that misuse is less likely and error effects are not catastrophic. In general, General Safety Design shall reflect applicable system and personnel safety factors, including the minimization of potential human error in the operation and maintenance of the system; be failure tolerant and eliminate or minimize possible hazards by removal of hazardous sources and operations by appropriate design measures or by prevention them from using safety devices or features. For designing a manufacturing workstation, it is necessary to obtain relevant information or data on task performance, equipment, working posture and environment. For this information we should turn to ergonomic guidance. The following ergonomical characteristics should be considered for work zone definition, proper equipment, tools, controls design, layout, task allocation:

- Body metrics (percentile of people's rate $5^{t h}, 50^{t h}, 95^{t h}$ ), optimal movement space and postures;

- Physical constrains/capabilities: strength, force characteristics, permissible level of noise, temperature, illumination, humidity, visual, auditory, tactual sensitivity, pain tolerance, fatigue, somatic characteristics, etc.

Taking into consideration robot structure we should know that forces, torques and velocities are different at each configuration. This means that robots can be very dangerous at one point and less perilous at others causing serious or moderate injuries to human working in the immediate vicinity. These facts should definitely be taken into account during task distribution and agents' mutual allocation if we want to enhance safety in our system. Design of robotic workstations should also be based on the analysis of the task requirements, time aspects, size of working area, robot's characteristics (DOF, speed, torque, inertia, angle of each joints, path, accuracy, range of movements, working envelope) dangerousity of each configuration should be estimated, interior, exterior facilities chosen (control architecture, sensors, visual system, AI, safety features), as well as human's roles in the system determined. The right combination of all these factors, techniques and nuances should be the starting point for designers whose tasks are chiefly oriented on human-centered work place design in robot space. Thus, human capabilities have to be investigated and defined in order to be implemented in system planning, as well as human weaknesses have to be detected and compensated.

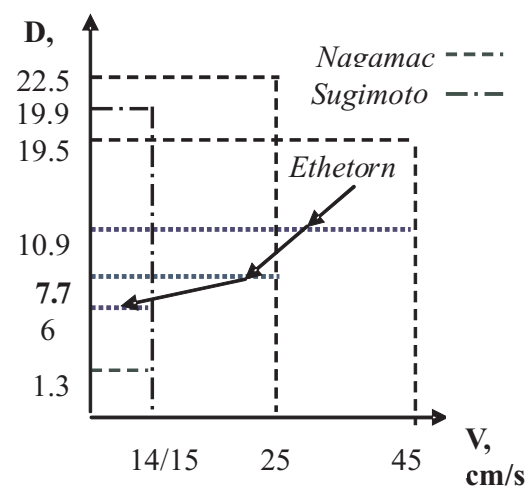

Fig. 5. Comparisons of results in safe distance evaluation.

\section{Goals and Future Work}

What we still need is to understand what kinds of robot movements are difficult to perceive, what can be done to enhance the perceptibility of the arm movement. We also need to provide experiments with common patterns of movements: PTP, linear movements, rotating, revolving, smooth movements and investigate human behavior at critical situations (risk taking actions). It is essential to provide the investigations and analyze the main parameters that enhance the visual, auditory and tactile human perception, define the optimal level of sensitivity and find the method to minimize the reaction time. After defining those magnitudes and providing series of experiments (simulations, self-report, behavioral measures) it is planned to build an augmented awareness system, where unwilling interactions and harmful consequences would be avoided. Currently we design a hybrid robotic cell (some examples of already exist implementations are displayed in Fig. 6), where robot operates within the human workspace improving, supporting and lightening his/her performance. From the ergonomic and safety point of view we examine activities related to human and robot interaction, using materials, instruments, postures, mutual allocation, trajectories of agents, task distribution, distances, duration of work, physical and mental workload, etc. We also perform the work flow analysis and make a risk assessment, considering possible human errors, robot failures, inappropriate allocations, etc. Based on those evaluations we propose the work cell where safety, efficiency, human's convenience and security would be enhanced. 
Fig. 6. Human Robot Disassembly cell [26], [27]
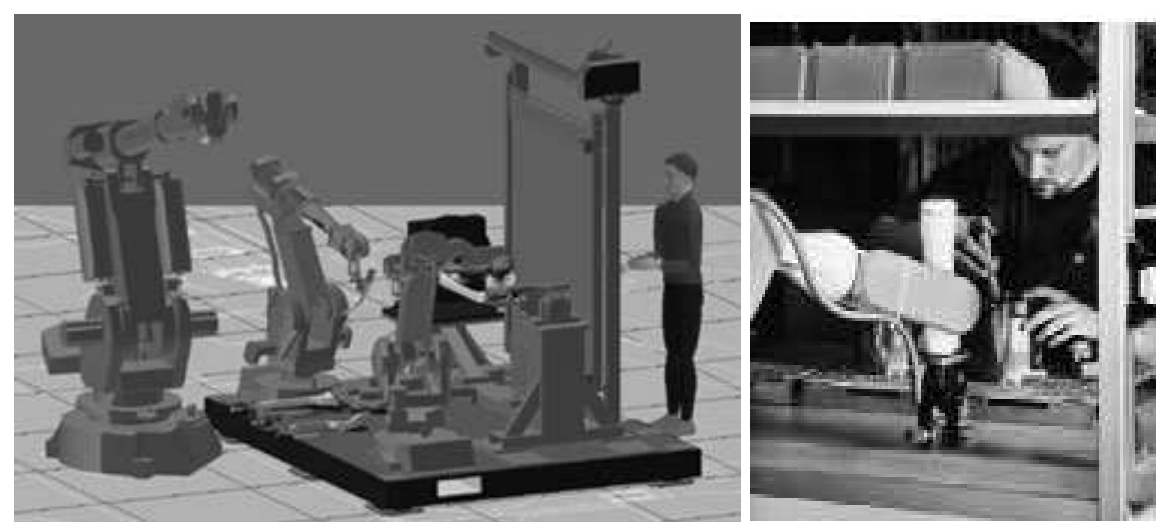

The maximum allowed distance between robot's work envelope and human work zone we defined as $20-30 \mathrm{~cm}$, robot's safe speed is $150 \mathrm{~mm} / \mathrm{s}$. This distance shouldn't be overlapped during the whole task performance. The position and the speed of the end effector are monitored by embedded position sensor and accelerometer. If the robot goes beyond the predefined trajectory or exceeds the speed, controller receive signal about failure and generate emergency stop signal. Human position and work place were obtained from the ergonomical considerations of $\mathrm{Hu}$ man factor in workplace design. Human's convenient reachability, strength, force distribution, anthropometrical parameters for the sitting posture were taking into account. Planning mutual allocation of agents and task distribution, we also looked at human's cognitive capabilities and sensory modality constrains. Video camera mounted on the ceiling above the workplace monitors the safe distance during operation. The color markers could be implemented for making the identification process of the human location with respect to the end-effector quicker and unambiguous. However, the question of safety and sensory system application is still open to question.

\section{Conclusion and Discussion}

In this article we have discussed only one side of the polyhedral model: human robot interaction. The human factor was considered as a major criterion, which takes significant rank in the whole system planning. It was shown that the role of cognitive engineering and ergonomics is becoming more and more important. With the advanced technology and development in robotics, the role of humans in the work system is changing from physical to mental activity. In such cases, the evaluation of mental fatigue, mental workload or decrement of vigilance and awareness must be carried out from the viewpoint of ergonomics and cognitive science so that the decrease of productivity, reliability, efficiency or the increase of hazardous situations or human errors due to inadequate workplace or interface design may be avoided. On the other hand, physical interaction with robots is becoming more desirable and essential nowadays, however, to provide this interaction in safe and favorable conditions for humans is still a big challenge that requires further consideration, with the human factor as the prime focus. Human-robot inter- action still needs to involve models of human problem solving behavior, models of human behavior in control tasks, displays including visual, auditory presentation, human work load and proficiency. The implications of all of these for a system level design of robotic systems are very significant and are among the most important research frontiers in robotic system studies. In addition the question of sensory system for providing safe performance in the hybrid work cell is still poorly developed and requires new approaches.

\section{References}

1 Gertman D, Blackman H, Human Reliability and Safety Analysis Data Handbook, John Willey\&Sons, New York, 1996.

2 Ikuta K, Nokata M, General Evaluation Method of Safety for Humancare Robots, Proceedings of the IEEE International Conference on Robotics, 1999.

3 Scholtz J, Jill L, Yanco A, Awareness in Human-Robot Interactions, IEEE Conference on Systems, Man and Cybernetics, 2003, DOI 10.1109/ROBOT.2004.1307409, (to appear in print).

4 Rahimi M, Karwowski W, A research paradigm in human-robot interaction., International Journal of Industrial Ergonomics 5 (1990), 59-71, DOI 10.1016/0169-8141(90)90028-Z. Elsevier.

5 Norman D, Cognitive engineering In User Centered System Design, Hillsdale, NJ, Erlbaum, 1986.

6 Reason J, Human error, Cambridge University, 1990.

7 McCormick E, Sanders M, Human Factors in Engineering and Design, McCraw-Hill, 1992.

8 Sanders M, McCormick E, Human Factors in Engineering and Design, New York, 1993. 7th Ed

9 Donald C, Vigilance. People in Control. Human Factors in Control Room Design, London: The Institution of Electrical Engineers, 2001.

10 Swain AD, Guttman HE, Handbook of Human Reliability Analysis with Emphasis on Nuclear Power Plant Applications, NUREG/ CR, 1983.

11 Gugerty L, Tirre W, Individual Differences in Situation Awareness (Mica R. Endsley, Daniel J. Garland, eds.), 2000.

12 Goodrich M, Olsen D, Crandall J, Palmer T, Experiments in Adjustable Autonomy, Proceedings of the IJCAI01 Workshop on Autonomy, Delegation, and Control: Interacting with Autonomous Agents, 2001.

13 Endsley MR, Direct Measurement of situation Awareness: Validity and Use of SAGAT, Situation Awareness Analsysis and Measurement, Lawrence Erlbaum Associates, address=Mahwah, New Jersey, 2000, pp. 173.

14 _ Theoretical Underpinnings of Situation Awareness: A Critical Review, Situation Awareness Analysis and Measurement, Erlbaum Associates, 2000 . 
15 Rasmussen J, Human Errors: A taxonomy for describing Human Malfunction in Industrial Installations (1981). RIS- M-2304 Roskilde.

16 Sugimoto N, Kawaguchi K, Fault Tree Analysis of Hazards Created by Robot, Proceedings of the 13th International Symposium on Industrial Robots and Robot, 1983, pp. 913-928.

17 Nof S, Handbook of industrial robotics, John Wiley \& Sons, New York, 1999.

18 Yamada Y, Evaluation of Human Pain Tolerance and Its Application to Designing Safety Robot Mechanisms for Human-Robot Coexistence, Journal of Robotics and Mechatronics 9 (1997), no. 1.

19 Griffin M, Vibration and motion, Hand book of Human Factors and Ergonomics, Wiley \& Sons, Canada, 2006.

20 Industrial Robots and Robot Systems: Safety Requirements, Washington. ANSI (b1999). ANSI/RIA R15.06.

21 Nagamachi M, Human Engineering Oriented Research on Industrial Robots, Human Engineering (1983).

22 Etherton J, Human factors in managing robot system downtime. In Success factors for implementing change: A manufacturing viewpoint, Society of ME, Michigan, 1988.

23 Nomura T, Kanda T, On Proposing the Concept of Robot Anxiety and Considering Measurement of It, Proceedings of the IEEE Internatonal Workshop on Robot and Human Interactive Communication, 2003, DOI 10.1109/ROMAN.2003.1251874, (to appear in print).

24 Bethell CL, Salomon K, Murphy RR, Burke JL, Survey of Psychophysiology Measurements Applied to Human-Robot Interaction, 16th IEEE International Conference on Robot \& Human Interactive Communication August 26-29, 2007 / Jeju, Korea.

25 Stork S, Stössel C, Müller HJ, Wiesbeck M, Zäh MF, Schubö A, $A$ Neuroergonomic Approach for the Investigation of Cognitive Processes in Interactive Assembly Environments, 16th IEEE International Conference on Robot \& Human Interactive Communication August 26-29, 2007 / Jeju, Korea.

26 available at http://www.cdcza.co.za/news250706.htm

27 available at http://www. care--o--bot.de 